\title{
Information-Theoretic Measures and Sequential Monte Carlo Methods for Detection of Regeneration Phenomena in the Degradation of Lithium-Ion Battery Cells
}

\author{
Marcos E. Orchard, Member, IEEE, Matías S. Lacalle, Benjamín E. Olivares, Jorge F. Silva, Member, IEEE,
} Rodrigo Palma-Behnke, Senior Member, IEEE, Pablo A. Estévez, Senior Member, IEEE, Bernardo Severino, Williams Calderon-Muñoz, and Marcelo Cortés-Carmona, Member, IEEE

\begin{abstract}
This paper analyses and compares the performance of a number of approaches implemented for the detection of capacity regeneration phenomena (measured in ampere-hours) in the degradation trend of energy storage devices, particularly Lithium-Ion battery cells. All implemented approaches are based on a combination of information-theoretic measures and sequential Monte Carlo methods for state estimation in nonlinear, non-Gaussian dynamic systems. Properties of information measures are conveniently used to quantify the impact of process measurements on the posterior probability density function of the state, assuming that sub-optimal Bayesian estimation algorithms (such as classic or risk-sensitive particle filters) are to be used to obtain an empirical representation of the system uncertainty. The proposed anomaly detection strategies are tested and evaluated both in terms of (i) detection time (early detection) and (ii) false alarm rates. Verification of detection schemes is performed using simulated data for battery State-Of-Health accelerated degradation tests, to ensure absolute knowledge on the time instant where a regeneration phenomenon occurs.
\end{abstract}

Index Terms-Capacity regeneration, information theoretic measures, lithium-ion battery, particle filters, state-of-health.

\section{ACRONYMS AND ABBREVIATIONS:}

FP

False Positive

KL Kullback-Leibler

MSE Mean Squared Error

$\mathrm{N}$

Negative detection result

Manuscript received January 15, 2014; revised June 02, 2014, September 02, 2014, October 07, 2014, and November 05, 2014; accepted January 04, 2015. Date of publication January 26, 2015; date of current version June 01, 2015. This work was supported by CONICYT under Project FONDECYT 1140774 and Innova-CORFO 12IDL2-16296. Associate Editor: E. Zio.

M. E. Orchard, J. F. Silva, and P. A. Estévez are with the Electrical Engineering Department, Universidad de Chile, Santiago, Chile and Advanced Mining Technology Center (AMTC), Universidad de Chile (e-mail: morchard@ing.uchile.cl).

M. S. Lacalle, B. E. Olivares, and R. Palma-Behnke are with the Electrical Engineering Department, Universidad de Chile, Santiago, Chile.

B. Severino is with the Center of Energy, Electrical Engineering Department, Universidad de Chile, Santiago, Chile.

W. Calderon-Muñoz is with the Mechanical Engineering Department, Universidad de Chile, Santiago, Chile.

M. Cortés-Carmona is with the Electrical Engineering Department, Universidad de Antofagasta, Antofagasta, Chile.

Digital Object Identifier 10.1109/TR.2015.2394356

$\begin{array}{ll}\text { P } & \text { Positive detection result } \\ \text { PDF } & \text { Probability Density Function } \\ \text { PF } & \text { Particle Filter } \\ \text { ROC } & \text { Receiver Operating Characteristic } \\ \text { RSPF } & \text { Risk-Sensitive Particle Filter } \\ \text { SOH } & \text { State-Of-Health } \\ \text { TP } & \text { True Positive } \\ \text { TN } & \text { True Negative }\end{array}$

NotATION:

$x_{1, k}$

$x_{2, k}$

$x_{3, k}$

$U_{k}$

$y_{k}$

$\delta(\cdot)$

$w_{k}^{(i)}$

$w_{k / k-1}^{(i)}$

$x_{k}^{(i)}$

$\pi_{k}\left(x_{k}\right)$

$p\left(x_{k} \mid y_{k}\right)$

$q\left(x_{k} \mid x_{k-1}\right)$

$H(p)$

$D_{\text {ent }}$

$D(p \| q)$
Estimate of the battery State-Of-Health at time $k$.

Estimate of minor differences with respect to the nominal degradation trend at time $k$.

Estimate, at time $k$, of the additional available State-Of-Health due to regeneration phenomena.

External input associated with the apparition of regeneration phenomena.

Measurement at time $k$.

Delta of Kronecker function.

Weight related to the $i^{\text {th }}$ particle in the posterior probability density at time $k$.

Weight related to the $i^{\text {th }}$ particle in the prior probability density at time $k$.

Realization of the state vector, associated with the $i^{\text {th }}$ particle at time $k$.

True state vector probability density function at time $k$.

Posterior probability density function of the state vector $x_{k}$.

Importance sampling distribution.

Entropy $H$ of a probability $p$ equipped with its probability mass function $p(x)$.

Entropy difference between posterior and prior probability density functions.

Kullback-Leibler divergence between probability distributions $q$ and $p$. 


\section{INTRODUCTION}

$\mathbf{T}$ HE development of optimal energy storage devices has been an issue of study in the main scientific communities around the world. This issue is a consequence of the exponential growth shown by the electric and hybrid vehicle industry, and the general proliferation of electronic devices. Regardless of the main purpose for which an energy storage device is needed, it is critical that battery management systems are able to determine in a reliable manner the battery State-Of-Health $(\mathrm{SOH})$, which is a measure that is associated to the degradation that the battery has suffered over the course of its life [1]. This task can only be accomplished through precise estimation algorithms that allow incorporating real online measurements of the process, and ambient variables, to determine the amount of remaining charge cycles. Online estimation algorithms, more specifically Sequential Monte Carlo methods such as Particle Filters (PFs) [2], [3], are especially suited to solve the aforementioned problem, given their capacity to combine the available system's measures of information and analytic or empiric models. However, some energy storage devices suffer from sudden, momentary, and occasionally considerable regeneration of their capacity. These changes, related to physicochemical aspects and temperature-loading conditions during charge and discharge cycles, are particularly important in the case of Lithium-Ion batteries because they often alter the trend of the $\mathrm{SOH}$ prediction curve, thus affecting the performance of estimation and prognosis modules based on Bayesian algorithms [3]. Here lies the importance of considering a detection module for these regeneration phenomena of the $\mathrm{SOH}$, which allows us to correctly isolate them in the $\mathrm{SOH}$ modelling.

This paper presents a solution to the aforementioned problem, which provides a framework capable of estimating the $\mathrm{SOH}$ while simultaneously detecting and isolating the effect of self-recharge phenomena through different detection techniques. The anomaly detection modules are based on a combination of PF-based state estimators and information-theoretic measures that allows detecting rare events within the evolution of the battery degradation process under analysis. The selection criteria for the best suitable detection module are based on the performance obtained when comparing type I vs. type II errors [3], considering the impact they have over the SOH estimation in the Mean Squared Error (MSE) sense. Frequency analysis approaches, such as Fourier based methods, are discarded due to the non-stationary, asynchronous, and random nature of the phenomenon to be analyzed.

\section{Problem Formulation}

Capacity regeneration in energy storage devices has been only briefly mentioned in literature [3]. Specifically in the case of Lithium-Ion batteries, this phenomenon has been represented as a self-charging in the logger, where certain operating conditions facilitate a sudden (and temporary) increment in the available capacity of the energy storage device at the next cycle (see Fig. 1(a)). These phenomena may significantly affect the accuracy and precision of $\mathrm{SOH}$ prognosis algorithms, if not properly isolated, because they directly alter both the initial condition and the uncertainty that are associated with the
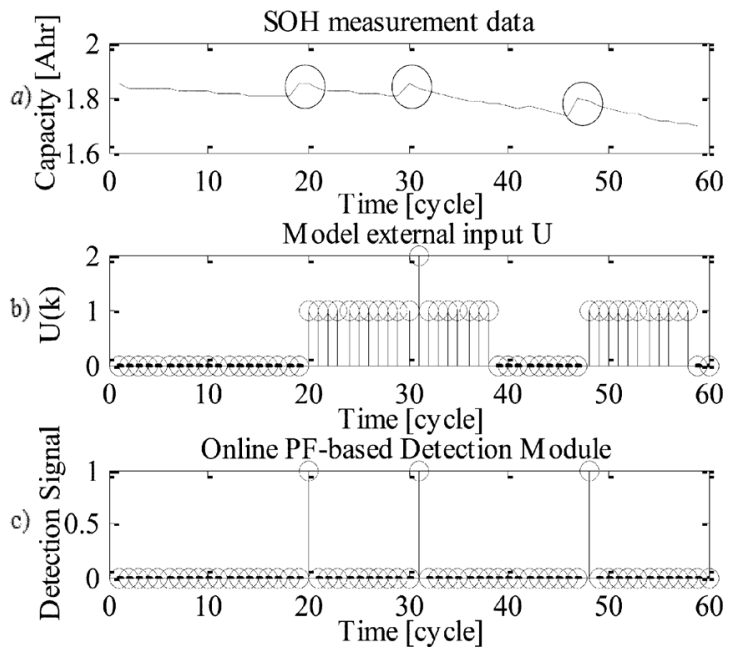

Fig. 1. a) Actual capacity degradation of a Lithium-Ion battery cell, where regeneration phenomena are conveniently marked; b) output signal $U$ from the detection module; and c) output of the PF-based capacity regeneration phenomena detector.

prediction stage [3], incorporating a bias in the estimate of the battery remaining useful life.

This paper analyses a solution for this problem considering an anomaly detection module that includes a PF-based SOH estimation framework; see Fig. 1(b), which illustrates the output of such a detection module when processing data as shown in Fig. 1(a). The main focus of this research is to compare several detection methods, determining their performance in terms of type I and type II errors, and their effect in the $\mathrm{SOH}$ estimation bias. All detection methods used in the design of hypothesis tests are based on PF algorithms, including classic and risk-sensitive versions [4]. These methods are complemented by the utilization of information-theoretic measures (e.g., entropy, mutual information, and divergence) to generate a detection indicator that characterizes the PF-based estimate of the state probability density function. We characterize the battery degradation process by the nonlinear state-space stochastic model (1)-(2) [3] because this particular model structure has proven to effectively combine empirical and phenomenological knowledge for a statistical characterization of self-recharge phenomena within the structure of the life cycle model.

\section{State transition model}

$$
\left\{\begin{array}{l}
x_{1, k+1}=\eta_{c} x_{1, k}+x_{2, k} x_{1, k}+\omega_{1, k} \\
x_{2, k+1}=x_{2, k}+\omega_{2, k} \\
x_{3, k+1}=\delta\left(U_{k}\right) \omega_{31, k}+\delta\left(1-U_{k}\right)\left(x_{3, k} \omega_{32, k}\right)+\ldots \\
\ldots \delta\left(2-U_{k}\right)\left(x_{3, k}+\omega_{31, k}\right)
\end{array}\right.
$$

\section{Measurement equation}

$$
y_{k}=x_{1, k}+\left(\delta\left(1-U_{k}\right)+\delta\left(2-U_{k}\right)\right) \cdot x_{3, k}+v_{k}
$$

$k$ is the cycle index, $x_{1}$ is a state representing the battery $\mathrm{SOH}$, $x_{2}$ is a state associated with an unknown model parameter that is required to explain minor differences with respect to the nominal degradation trend $\eta_{c}$ (for example manufacturing defects that are specific to each Lithium-Ion battery cell), $x_{3}$ is a state associated with the additional available $\mathrm{SOH}$ due to regeneration phenomena, and $y_{k}$ is the measured $\mathrm{SOH}$. Noises $\omega_{1}, \omega_{2}$, 
$\omega_{31}, \omega_{32}$, and $v$ are non-Gaussian sources of uncertainty, and $U$ is an external input associated with the apparition of regeneration phenomena. More specifically, the external system input $U$ is defined as a ternary signal, computed as a function of the output of online PF-based detection modules [5]:

$$
U(k)= \begin{cases}0 & \begin{array}{l}
\text { if self-recharge does not exist. } \\
1
\end{array} \\
\text { if either self-recharge is detected at cycle } k, \\
\text { or self-recharge phenomenon is fading. } \\
2 & \begin{array}{l}
\text { if additional self-recharge phenomena are } \\
\text { detected before the latest one fades. }
\end{array}\end{cases}
$$

Thus, the implementation of different strategies for the detection of regeneration phenomena also affects the performance of the state estimator.

The state transition model (1) \& (2) enables the implementation of Bayesian filtering techniques to monitor degradation processes in Lithium-Ion batteries, but it requires the implementation of an efficient detection module for the detection of regeneration phenomena. This fact motivates the development of anomaly detection modules, either based on PF-algorithms as in [5], [6], or information-theoretic measures [7]. This module performs a test for a null hypothesis that affirms that self-recharge phenomena either do not exist or are fading in time. The framework considers a PF-based state estimator for model (1) \& (2) that uses the output of the detection stage, where input $U$ is used to indicate if a regeneration phenomenon has occurred. The selected detection measure must enable isolating events where the $\mathrm{SOH}$ estimation algorithm is unable to track the process measurements using a predetermined state transition model. The main aspects associated to the formulation of this type of detection modules are described next, in Section III.

\section{Anomaly Detection Based on Particle FILTERING ALGORITHMS}

Nonlinear filtering is defined as the process of using noisy observation data $Y=\left\{y_{k}, K \in N\right\}$ to estimate at least the first two moments of a state vector $X=\left\{x_{k}, k \in N\right\}$ governed by a dynamic, nonlinear, non-Gaussian state-space model [7]. From a Bayesian standpoint, filtering procedures intend to generate an estimate of the posterior probability density function $p\left(x_{k} \mid y_{1: k}\right)$ for the state, based on the set of received measurements. Particle Filters are a class of algorithms that intend to solve this estimation problem by efficiently selecting a set of $N$ $\gg 1$ particles $\left\{x_{k}^{(i)}\right\}_{i=1 \cdots N}$, and weights $\left\{w_{k}^{(i)}\right\}_{i=1 \cdots N}$, such that the state Probability Density Function (PDF) may be approximated [8] by the empirical distribution

$$
\tilde{\pi}_{k}^{N}\left(x_{k}\right)=\sum_{i=1}^{N} w_{k}^{(i)} \delta\left(x_{k}-x_{k}^{(i)}\right) ;
$$

and the values of the particles weights $w_{k}^{(i)} \propto w\left(x_{k}^{(i)}\right)$ can be computed by

$$
w\left(x_{k}^{(i)}\right)=w_{k-1}^{(i)} \cdot \frac{p\left(y_{k} \mid x_{k}^{(i)}\right) p\left(x_{k}^{(i)} \mid x_{k-1}^{(i)}\right)}{q_{k}\left(x_{k}^{(i)} \mid x_{k-1}^{(i)}\right)} \forall i \in\{1, \ldots, N\}
$$

where $q_{k}\left(x_{k}\right)$ denotes the importance sampling density function [9]. The choice of this importance density function $q_{k}\left(x_{k}\right)$ is critical for the performance of the particle filter scheme. In the particular case of classical PF, the value of the particle weights $w_{k}^{(i)}$ is computed by setting the importance density function equal to the prior PDF for the state, i.e., $q_{k}\left(x_{k} \mid x_{k-1}\right)=p\left(x_{k} \mid x_{k-1}\right)$ [9]. Although this choice of importance density is appropriate for estimating the most likely probability distribution according to a particular set of measurement data, it does not offer a good estimate of the probability of events associated to high-risk conditions and low likelihood. Indeed, the Risk-Sensitive Particle Filter (RSPF) seeks to solve this issue.

This paper uses the PF- and RSPF-based state PDF estimates to implement detection modules. In addition, it explores the possibility of using information-theoretic measures to analyze those estimates, to perform early detection of $\mathrm{SOH}$ regeneration phenomena that may take place in energy storage devices.

\section{A. Anomaly Detection Based on Classic Particle Filtering Algorithms}

PF-based anomaly detection modules [5]-[7], [10], [11] have been used in the past to identify abnormal conditions in nonlinear, non-Gaussian dynamic systems. The objective in this type of implementations is to fuse the information that is available at a feature vector (measurements) to generate estimates of the prior state PDF that could be helpful when determining either the operating condition (mode) of a system or deviations from desired behavioral patterns. This compromise between model-based and data-driven techniques is accomplished by the use of a PF-based module built upon the nonlinear dynamic state model. PF-based detection modules provide a framework where customer specifications (such as false alarm rate and desired probability of detection) can be easily managed and incorporated within the algorithm design parameters.

This paper uses the PF-based detection module presented in [3] as the baseline to compare other detection approaches. This module applies a hypothesis testing procedure, where the a priori PF-based PDF estimate at time $k$ is used to compute a time-varying threshold $T(k)$, which is defined as the largest scalar such that the sum of the weights $w_{k}^{(i)}$, for all particles that satisfy $x_{k}^{(i)} \geq T(k)$, is greater than the desired false alarm rate $\alpha \%$ [5]. Therefore, the threshold at each time cycle $k$ depends on both the PF-based PDF estimate and the false alarm rate. Fig. 2 illustrates this point. As a result, the detection module performs a hypothesis test (with a false alarm rate $\alpha \%$ determined by the user) for the measurement $y_{k}$, considering the prior one-step ahead prediction of the system output as the PDF that characterizes the null hypothesis (the self-recharge phenomena either does not exist or is fading in time). In other words, if the null hypothesis is rejected at cycle $k$, it means that the measurement $y_{k}$ is larger than the detection threshold (for $\alpha \%$ statistical confidence) that is computed from the predicted (one-step) state PDF.

\section{B. Anomaly Detection Based on Risk-Sensitive Particle Filtering}

The problem of early detection using PF-based approaches has been discussed in [12]. The method therein proposed uses a RSPF framework to represent the probability of rare events (in this particular case, the capacity regeneration phenomena 


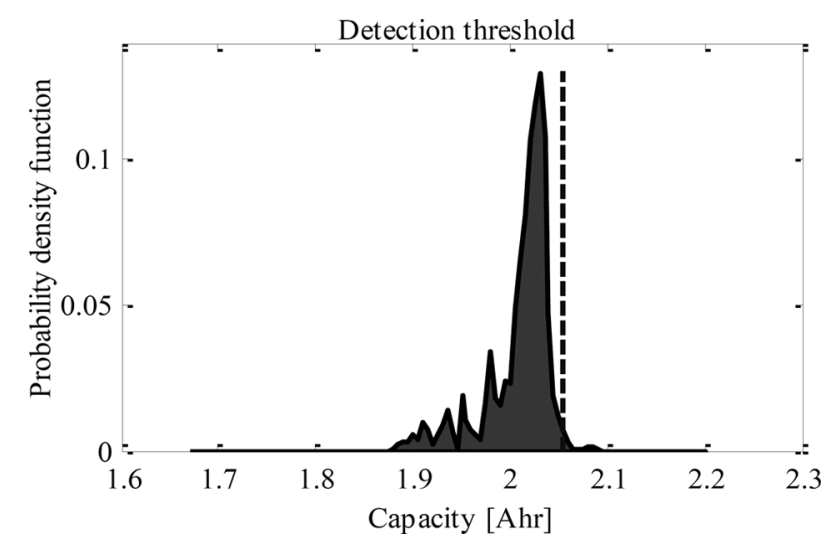

Fig. 2. Illustration of a time-varying threshold that is computed from a priori PF-based PDF estimates.

in energy storage devices) within the formulation of an importance density function that aims at generating more particles in high-risk regions of the state-space. However, [12] only provided a qualitative analysis of the trend in a problem of model parameter estimation, without establishing a formal procedure to implement a decision-making process.

Mathematically, RSPF considers an importance distribution that is set as

$$
q\left(d_{k}, x_{k} \mid d_{k-1}^{(i)}, x_{k-1}^{(i)}, y_{1: k}\right)=\gamma_{k} \cdot r\left(d_{k}\right) \cdot p\left(d_{k}, x_{k} \mid y_{k}\right)
$$

where $d_{k}$ is a set of discrete-valued states representing a fault mode, $x_{k}$ is a set of continuous-valued states that describe the evolution of the system given those operating conditions, $r\left(d_{k}\right)$ is a positive risk function that is dependent on the fault mode, and $\gamma_{k}$ is a normalizing constant. Thus, a RSPF-based detection module should define a risk importance sampling distribution that ensures the existence of particles in the tails of the state PDF, to help represent the event of unlikely regeneration phenomena. In situations where effectively the data show no signs of these events, the weights of the particles located at the tails of the PDF should decrease over time. The formulation of the hypothesis test and its corresponding threshold is similar to what has been already discussed in the case of PF-based detection modules.

The use of information-theoretic measures for the implementation anomaly detection modules is motivated by the assumption that any anomaly should qualitatively affect the PDF estimates. In this regard, the following section focuses on the most important concepts that need to be taken into account when implementing these measures (in this case, entropy) to analyze and characterize sampled versions of the posterior distribution.

\section{INFORMATION-THEORETIC MEASURES FOR PARTICLE FILTERING ESTIMATES}

Several examples that incorporate information-theoretic measures to analyze the outputs of particle filtering algorithms can be found in [13]-[16]. This article aims at the formulation of anomaly detection modules based on PF, which are combined with the use of information measures, particularly focused on the widely known information indicators like Shannon entropy [17], and Kullback-Leibler (KL) divergence.

\section{A. Entropy}

Entropy is a measure of uncertainty for a discrete random variable that is associated to its discrete probability measure. In particular, the entropy $H$ of a probability $p$ equipped with its probability mass function $p(x)$ is given by

$$
H(p)=-\sum_{x \in \chi} p(x) \log (p(x)) .
$$

Few additional considerations are required when trying to compute the entropy in the case of PF-based estimates of the conditional state PDF. Indeed, the entropy of PF-based estimates of the posterior state PDF, given a set of measurements $y_{1}, \ldots, y_{\mathrm{k}}$, is computed as shown in (7) [18] at the bottom of the page, where $w_{k-1 / k-1}^{(i)}=w_{k-1}^{(i)}$ and $w_{k / k-1}^{(i)}$ are the particle weights of the prior PDF at time $k$, and $N$ is the number of particles. The latter expression will be of use when evaluating the uncertainty associated to online estimates in dynamic processes. More details can be found in [18].

Entropy-related applications for PF algorithms generally aim at evaluating how many statistically independent identically distributed samples the filtering algorithm requires to represent regions of the state space that accumulate the majority of the probability mass, for a given state PDF estimate $p(x)$. This approach is useful because the entropy indicator should remain stable around a particular value while no regenerations of the $\mathrm{SOH}$ are detected. On the contrary, the entropy should suddenly increase when these phenomena are present, allowing us to set a threshold to determine if an increment of the $\mathrm{SOH}$ is detected as a regeneration of its capacity. In this regard, the proposed anomaly detection scheme uses the fact that any sudden abnormal condition in the battery degradation process should affect the distribution of the PF-based posterior state estimate. This effect on the posterior estimate is caused by the fact that,

$$
\begin{aligned}
H\left(p\left(x_{k} \mid y_{k}\right)\right)= & \log \left(\sum_{i=1}^{N} w_{k / k-1}^{(i)} p\left(y_{k} \mid x_{k / k-1}^{(i)}\right)\right) \\
& -\sum_{j=1}^{N} w_{k / k}^{(j)}\left[\log \left(p\left(y_{k} \mid x_{k}^{(j)}\right)\right)+\log \left(\sum_{i=1}^{N} w_{k-1 / k-1}^{(i)} p\left(x_{k}^{(j)} \mid x_{k-1}^{(i)}\right)\right)\right]
\end{aligned}
$$


under abnormal operating conditions, the system model no longer represents the best choice for the importance sampling distribution [19]. As a consequence, the weights associated to particles with low-likelihood undergo strong corrections, increasing the entropy of the conditional state PDF.

\section{B. Entropy Difference}

Another anomaly detection method that we have included in the analysis corresponds to the entropy difference. In this scheme, the discrete entropy is calculated for both the prior and the posterior state PDF estimates. The detection measure is obtained by subtracting the entropy of the posterior state PDF $p\left(x_{k} \mid y_{k}\right)$ to the entropy of the prior state PDF $p\left(x_{k} \mid x_{k-1}\right)$, as in (8).

$$
D_{\text {ent }}=H\left(p\left(x_{k} \mid x_{k-1}\right)\right)-H\left(p\left(x_{k} \mid y_{k}\right)\right) .
$$

This measure uses the fact that the entropy is at a maximum when all the particle weights are equal, and conversely is minimal when the particle population has degenerated [5] (i.e., one particle has a weight equal to one). As a result, the entropy of the posterior PDF should decrease, with respect to the value associated with the prior PDF estimate, when only a reduced number of particles offer high likelihood to the measured data, as in the case of unexpected capacity regeneration phenomena in Lithium-Ion batteries. Thus, when this event occurs, the entropy difference measure should increase. If an appropriate threshold is selected, this measure can be used to detect the moment when a specific increment in the $\mathrm{SOH}$ value is related to a capacity regeneration event.

\section{Kullback-leibler Divergence}

The KL divergence $D(p \| q)$ is an information-theoretic measure that quantifies the inefficiency associated with assuming that a random variable $x$ has a probability distribution $q(x)$, when the true PDF is $p(x)$ [17]. The KL divergence for discrete distributions is defined as

$$
D(p(x) \| q(x))=\sum_{x \in \chi} p(x) \log \left(\frac{p(x)}{q(x)}\right) .
$$

An anomaly detection module based on this measure considers how much information is gained by updating the weights of the prior distribution to generate the posterior PDF state estimate, and using for this purpose recently acquired measurements. Considering this idea, the Kullback-Leibler divergence can be used as in (10).

$$
\begin{aligned}
& D\left(p\left(x_{k} \mid y_{k}\right) \| p\left(x_{k} \mid x_{k-1}\right)\right) \\
& \quad=\sum_{x \in \chi} p\left(x_{k} \mid y_{k}\right) \log \left(\frac{p\left(x_{k} \mid y_{k}\right)}{p\left(x_{k} \mid x_{k-1}\right)}\right) .
\end{aligned}
$$

In the implementation of detection modules based on this measure, a one-step PF-based estimate of the prior state PDF is computed assuming that regeneration phenomenon have not occurred. If this assumption is valid, the posterior state PDF should differ minimally from the prior, and thus the Kullback-Leibler divergence between both PDFs should provide a value close to zero. Conversely, if a capacity regeneration event occurs, then the difference between the two PDF estimates (prior and posterior) should be significant, thus helping to detect the phenomenon of interest by using a pre-determined threshold.

\section{Anomaly Detection Modules Based ON INFORMATION-THEORETIC MEASURES AND PARTICLE FILTERING AlgORITHMS}

Self-recharge phenomena detection in Lithium-Ion batteries has been selected as a case study to compare the different aforementioned detection modules. Verification of the proposed detection schemes is performed using simulated data for $\mathrm{SOH}$ accelerated degradation tests, and following the method proposed in [3]. Simulated data are required to ensure absolute knowledge on the time instant where a regeneration phenomenon occurs. Performance is evaluated by computing several realizations of the stochastic process that defines each one of the proposed PF-based detection modules, and estimating the type I and II errors. Also, the impact that each detection scheme has on the Bayesian processor that estimates the battery $\mathrm{SOH}$ is measured in terms of the mean-squared error MSE for the posterior estimate, precision, and detection accuracy indices. Also, the impact that each detection scheme has on the Bayesian processor that estimates the battery $\mathrm{SOH}$ is measured in terms of the MSE:

$$
M S E=\frac{1}{N} \sum_{i=1}^{N}\left(y_{i}-\hat{y}_{i}\right)^{2},
$$

where $N$ is the number of battery $\mathrm{SOH}$ measurements, $y_{\mathrm{i}}$ corresponds to the actual battery $\mathrm{SOH}$ measured at time $i$, and $\hat{y}_{i}$ is the posterior $\mathrm{SOH}$ estimate.

The precision index is defined as the ratio between the number of correctly detected events, also known as the True Positives (TPs), and the total number of detections performed. The total number of detections is computed as the sum of the TPs and False Positives (FPs). The precision index is a measure that helps to determine the veracity of a result that has been classified as positive. The expression for the precision is

$$
\operatorname{Prec}=\frac{T P}{(T P+F P)} .
$$

The accuracy index, on the other hand, is computed as the ratio between all correctly classified results and the total number of classified results. The former is computed as the sum of the TPs and True Negatives (TNs), while the latter is the sum of all Positive $(\mathrm{P})$ and Negative $(\mathrm{N})$ detection results.

$$
\text { Acc }=\frac{(T P+T N)}{(P+N)}
$$

As previously mentioned, the analysis considered 10 different simulated data sets to properly capture the behavior of an accelerated degradation process for Lithium-Ion batteries. The use of simulated data is necessary to reliably compute the number of true and false positives, provided that there is absolute knowledge about the time where regeneration phenomena occur. Nevertheless, the presented methods can also be applied to actual degradation data.

This section presents the results obtained after the implementation of the detection methods described in Sections III and 


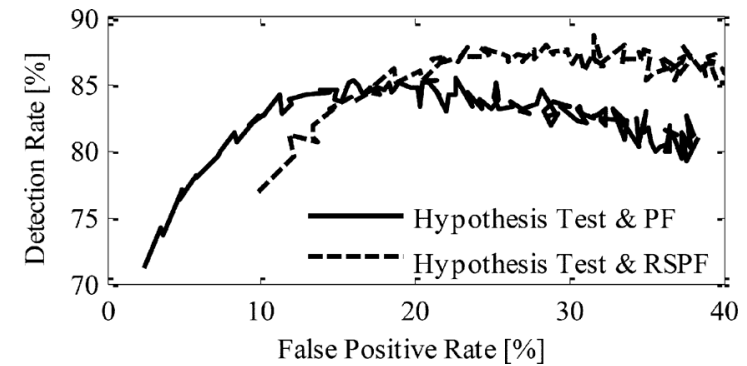

Fig. 3. ROC curve for detection modules based on PF- and RSPF-based hypothesis testing procedures.

IV. First, hypothesis testing procedures based on classic and risk-sensitive [3] versions of PF algorithms are shown. Second, the emphasis is focused on detection modules based on information-theoretic measures, and both the classic version and risksensitive versions of PF algorithms [19]. Considering the stochastic nature of particle filtering algorithms, a fair comparison between all detection schemes cannot be made in terms of just one realization. For this reason, for each one of the 10 aforementioned data sets, 40 realizations of the PF estimator were computed. Thus, each data point in Fig. 3 through Fig. 9 averages the performance of the detector over 400 realizations of the state estimator.

\section{A. Hypothesis Testing Procedures}

Hypothesis testing procedures assume that the posterior state PDF for the previous time instant $k-1$ is computed by using the classic version of the PF algorithm and model (1) \& (2). Nevertheless, two different implementations of anomaly detection modules are hereby compared. On the one hand, the method denominated as Hypothesis Test \& PF uses the classic PF algorithm to generate the prior state PDF within the detection module at time $k$. On the other hand, the method denominated as Hypothesis Test \& RSPF uses a RSPF algorithm to generate the aforementioned prior PDF. Both approaches perform the procedure described in Section III, using the prior PDF estimates as null hypotheses for the $\mathrm{SOH}$ measurement $y_{\mathrm{k}}$. Fig. 3 shows the Receiver Operating Characteristic (ROC) curves for each one of the proposed detection schemes, characterizing their performance in terms of the false alarm rate $(\alpha)$, and the detection probability $(\beta)$.

The PF-based detection module shows better performance than the RSPF-based module for values of $\alpha$ under $17 \%$. For values above that threshold, the RSPF algorithm improves its performance against the classic PF algorithm, achieving better detection rates $\beta$ for the same values of $\alpha$. These results could help to promote the idea of choosing Hypothesis Test \& RSPF as the most adequate method because the model (1) \& (2) can easily compensate the effect of false positives through an adequate estimation of the state $x_{3}$, while misdetections could represent an important issue for the implementation of $\mathrm{SOH}$ prognosticators (because $U_{k}=0$ implies that $y_{k}=x_{1, k}+v_{k}$, and thus the estimate of state $x_{1}$ will suffer a significant, but unnecessary, update) [3]. However, it should be also noted that RSPF tends to increase the bias of the PDF estimate as the detection rate (and the false positives rate) increases. However, the MSE

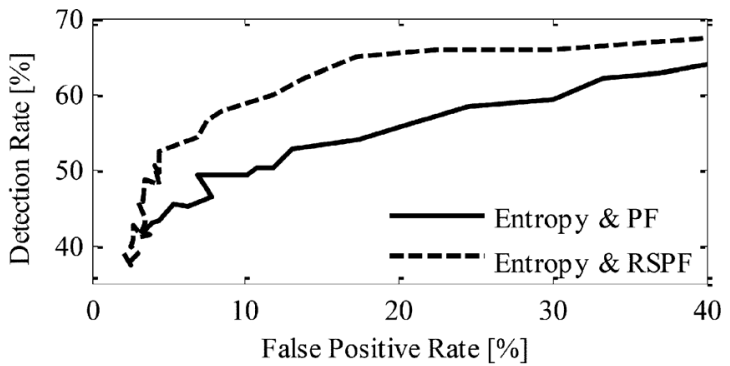

Fig. 4. ROC curve for detection modules based on entropy of the posterior PDF estimate.

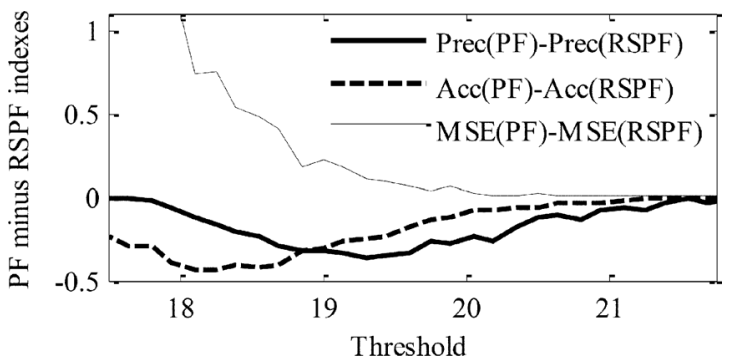

Fig. 5. Difference between performance indices related to Entropy \& $P F$ and Entropy \& RSPF methods.

obtained by the two approaches shows a negligible difference (on the order of 1e-5) due to the fast adaptation of the samples in methods of sequential Monte Carlo. This result confirms that, for higher detection rates, the Hypothesis Test \& RSPF approach has better performance.

\section{B. Theoretical Information Measures}

1) Entropy of the Posterior PDF Estimate: We implemented two anomaly detection approaches based on a threshold for the entropy of posterior PDF estimates. Similar to what was described in Section V-A, the posterior state PDF for the previous time instant $k-1$ is computed by using the classic version of the PF algorithm and model (1) \& (2). On the one hand, Entropy $\& P F$ uses the classic $\mathrm{PF}$ algorithm to compute the posterior state PDF at time $k$, whereas Entropy \& RSPF utilizes a RSPF version. Fig. 4 shows the ROC curves for each one of these entropy-based detection modules.

Fig. 4 shows better performance for the Entropy \& RSPF scheme because it provides improved detection probability for any given false positive rate. These results are justified by the fact that the RSPF algorithm allocates samples in areas of the state space that are related to high-risk events, such as $\mathrm{SOH}$ regeneration phenomena, thus easing the incorporation of these events in the posterior PDF.

To quantitatively confirm this statement, Fig. 5 shows the difference between precision, accuracy, and MSE indicators for Entropy \& PF versus Entropy \& RSPF for a range of entropy detection thresholds. The difference between the aforementioned indicators for both approaches shows that Entropy \& $R S P F$ obtains higher values than Entropy $\& P F$, both in terms of precision and accuracy in detection.

Furthermore, if the detection module uses Entropy \& $R S P F$ to generate the input signal $U_{\mathrm{k}}$ in model (1) \& (2), then the battery 


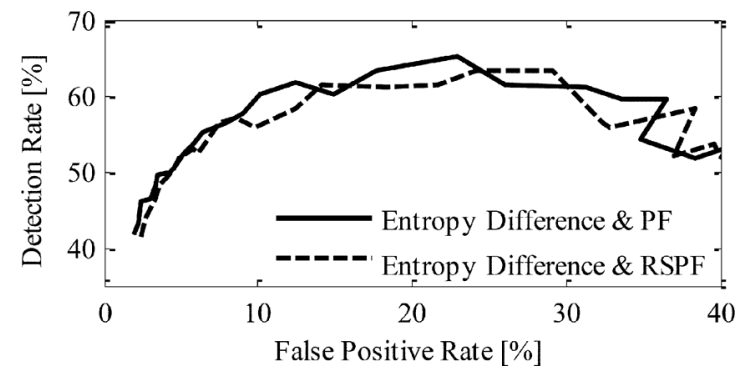

Fig. 6. ROC curve for detection modules based on the difference of entropy between prior and posterior PDF estimates.

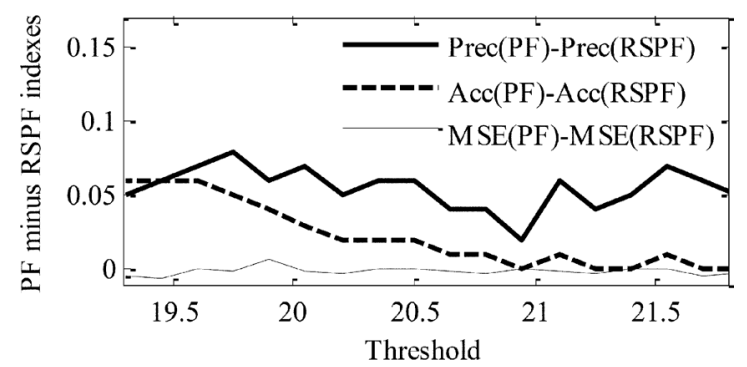

Fig. 7. Difference between performance indices related to Entropy Difference $\& P F$ and Entropy Difference \& RSPF methods.

SOH posterior estimate exhibits lower MSE with respect to the ground truth $\mathrm{SOH}$ than in the case of Entropy \& PF. As an additional outcome of this analysis, Fig. 5 also provides useful information to determine the most appropriate threshold to maximize the precision and accuracy of the detector module as deemed appropriate.

2) Entropy Difference Between Prior and Posterior PDF Estimates: Two approaches implemented for the detection of battery $\mathrm{SOH}$ regeneration use the difference between the prior and posterior state PDF estimates. On the one hand, Entropy Difference \& $P F$ utilizes the classic PF algorithm to estimate each one of the aforementioned distributions. On the other hand, Entropy Difference \& RSPF uses a RSPF version. Results, in terms of type I and type II errors for both approaches, are presented in Fig. 6.

According to what Fig. 6 shows, ROC curves for both detection modules are quite similar. The same conclusions can be made when analyzing the difference between precision, accuracy, and MSE indicators for a range of entropy detection thresholds, as shown in Fig. 7.

Fig. 7 allows us to distinguish slight differences between PFand RSPF-based algorithms. In terms of precision and accuracy, the use of the classic PF exhibits a small advantage over RSPF. However, regarding the MSE of the posterior battery SOH estimate that is obtained when these methods are used to generate the input signal $U_{k}$ in model (1) \& (2), it can be seen that there are no significant differences between these two approaches.

3) Kullback-Leibler Divergence: Finally, two approaches were implemented for detection of battery $\mathrm{SOH}$ regeneration phenomena based on the Kullback-Leibler divergence between prior and posterior state PDF estimates. KL-Divergence \& PF utilizes the classic PF algorithm to estimate each one of the aforementioned distributions, while KL-Divergence \& $R S P F$

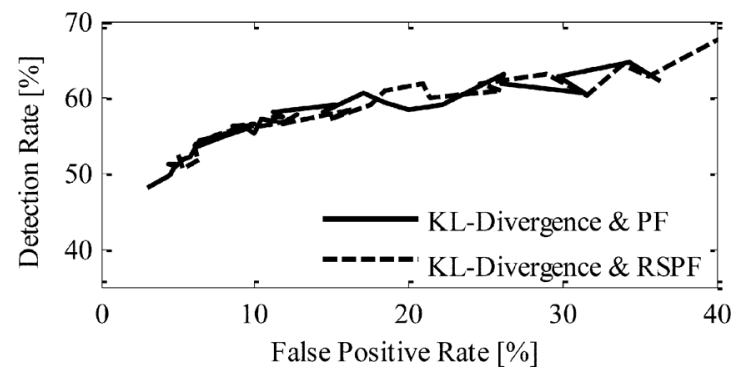

Fig. 8. ROC curve for detection modules based on KL Divergence.

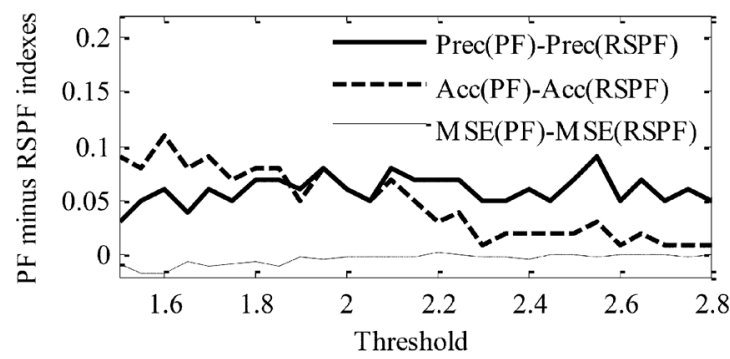

Fig. 9. Difference between performance indices related to $K L$-Divergence \& $P F$ and $K L$-Divergence \& $R S P F$ methods.

uses a RSPF version. Results, in terms of type I and type II errors for both approaches, are presented in Fig. 8.

Fig. 8 shows that, corresponding to what happened in the case of entropy difference, the ROC curves exhibit comparable results for both algorithms. Thus, the analysis of performance indices (see Fig. 9) is central when comparing the two approaches.

In this regard, Fig. 9 shows that both accuracy and precision indices for $K L$-Divergence \& $P F$ are better than the ones obtained by the $K L$-Divergence \& $R S P F$ detection module. The MSE presents similar behavior in both cases, being slightly lower for $K L$-Divergence \& $P F$, which leads to the conclusion that the PF-based algorithm performs better than its RSPF counterpart in this case.

\section{Performance Analysis of the Proposed Detection MODULES FOR BATTERY SOH REGENERATION}

The main concept behind the proposed anomaly detection schemes is that any sudden abnormal behavior in the system should affect the posterior PDF estimate that is computed by the Bayesian processor. This idea is based on the fact that, under abnormal operating conditions, the system model cannot be appropriately represented by the proposed importance sampling distribution. In this regard, the methods that were presented in this work offer reasonable performance in terms of their ability to detect capacity regeneration phenomena. However, a more in-depth analysis of the results obtained in terms of the ROC curves, accuracy, and precision indices allows us to make some distinctions.

All methods based on information-theoretic measures exhibit similar performance in terms of their ROC curves (see Figs. 4, 6 , and 8). It is interesting to note that the RSPF-based entropy method outperforms other measures of information, particularly in areas with higher detection rates, due to a better characterization of high-risk events (such as regeneration phenomena) 
within the importance sampling distribution that this method utilizes. As for the detection modules that are based on hypothesis testing procedures, both alternatives (PF- or RSPF-based) perform notoriously better than the one based on information theoretic measures, achieving a higher detection rate for every value of $\alpha$ (false positive rate). These results are consistent with the intuitive idea that a hypothesis test in this case is the closest alternative to a Neyman-Pearson test, which would be the optimal decision rule for a simple two-class decision problem. Depending on the desired rate of false positive, the user can choose between a module based on the classic PF algorithm or a RSPF version. While the first approach achieves better performance for lower rates of false positives, it is important to consider that the RSPF-based detection modules show improvements over the classic PF scheme, in terms of maximum probability of detection. Evidence, in this case, supports this statement mainly because these approaches incorporate the regeneration phenomena effect in the importance sampling distribution, allowing better detection rates and early detection. Given that prognostic methods based on model (1) \& (2) are unaffected by false positives, but highly sensitive to the misdetection of regeneration phenomena due to the state $x_{3}$, [3], we recommend the implementation of RSPF.

Regarding the selection of the detection module that shows a better performance considering measures of precision and accuracy, it is not possible to reach a conclusive answer. In terms of accuracy, the results of the Kullback-Leibler divergence between prior and posterior state PDF estimates show a slight improvement when using the classic version of PF algorithms.

\section{CONCLUSION}

This paper presented the development and evaluation of different methods for the detection of capacity regeneration phenomena in Lithium-Ion battery cells. A total of eight different methods were implemented, considering different measures of the quality of state PDF estimates, and two versions of sequential Monte Carlo algorithms: classic PF, and RSPF. A throughout performance analysis that considered type I and type II detection errors, precision, detection accuracy, and MSE of the state estimator was conducted to evaluate the contribution of information-measurement-based detectors with respect to hypothesis testing procedures based on both the classic PF and risk-sensitive algorithms.

From the obtained results, we surmise that the implementation of hypothesis testing procedures outperforms informationmeasurement-based approaches for detection of capacity regeneration, although the choice of classic PF algorithms versus risk-sensitive variants depends on the desired probability of detection. The latter, because a detector that computes a fault indicator, from the posterior state PDF estimate, is more capable of isolating regeneration phenomena in the degradation curve if the a priori PDF incorporates those phenomena in the importance sampling distribution.

Future work will focus on the implementation of NeymanPearson tests based on a proper characterization of the probability distribution associated with capacity regeneration phenomena. Although this is a very complex task, we believe it could help to significantly increase the probability of detection of these events through the implementation of a simple two-class hypothesis testing procedure.

\section{REFERENCES}

[1] B. Pattipati, K. Pattipati, J. P. Christopherson, S. M. Namburu, D. V. Prokhorov, and L. Qiao, "Automotive battery management systems," in IEEE AUTOTESTCON 2008 Conf. Record, Salt Lake City, UT, USA, Sep. 8-11, 2008, pp. 581-586.

[2] B. Saha, K. Goebel, S. Poll, and J. Christophersen, "Prognostics methods for battery health monitoring using a Bayesian framework," IEEE Trans. Instrum. Meas., vol. 58, no. 2, pp. 291-296, Feb. 2009.

[3] B. Olivares, M. Cerda, M. Orchard, and J. Silva, "Particle-filtering-based prognosis framework for energy storage devices with a statistical characterization of state-of-health regeneration phenomena," IEEE Trans. Instrum. Meas., vol. 62, no. 2, pp. 364-376, Feb. 2013.

[4] M. Orchard, L. Tang, B. Saha, K. Goebel, and G. Vachtsevanos, "Risksensitive particle-filtering-based prognosis framework for estimation of remaining useful life in energy storage devices," Stud. Informat. Control, vol. 19, no. 3, pp. 209-218, Sep. 2010.

[5] M. Orchard and G. Vachtsevanos, "A particle filtering approach for on-line fault diagnosis and failure prognosis," Trans. Inst. Meas. Control, vol. 31, no. 3-4, pp. 221-246, Jun. 2009.

[6] M. Orchard, L. Tang, and G. Vachtsevanos, "A combined anomaly detection and failure prognosis approach for estimation of remaining useful life in energy storage devices," in Proc. Annu. Conf. Prognostics and Health Manage. Soc., Montreal, QC, Canada, 2011.

[7] M. Orchard, B. Olivares, M. Cerda, and J. Silva, "Anomaly detection based on information-theoretic measures and particle filtering algorithms," in Proc. Annu. Conf. Prognostics and Health Manage. Soc., Minneapolis, MN, USA, 2012.

[8] A. Doucet, N. de Freitas, and N. Gordon, "An introduction to sequentia Monte Carlo methods," in Sequential Monte Carlo Methods in Practice, A. Doucet, N. de Freitas, and N. Gordon, Eds. New York, NY, USA: Springer-Verlag, 2001.

[9] M. S. Arulampalam, S. Maskell, N. Gordon, and T. Clapp, "A tutorial on particle filters for online nonlinear/non-Gaussian Bayesian tracking," IEEE Trans. Signal Process., vol. 50, pp. 174-188, 2002.

[10] V. Verma, G. Gordon, R. Simmons, and S. Thrun, "Particle filters for rover fault diagnosis," IEEE Robot. Autom. Mag., pp. 56-64, 2004.

[11] B. Zhang, C. Sconyers, C. Byington, R. Patrick, M. Orchard, and G. Vachtsevanos, "A probabilistic fault detection approach: Application to bearing fault detection," IEEE Trans. Ind. Electron., vol. 58, no. 5, pp. 2011-2018, May 2011.

[12] M. Orchard, G. Kacprzynski, K. Goebel, B. Saha, and G. Vachtsevanos, "Advances in uncertainty representation and management for particle filtering applied to prognostics," in Proc. Int. Conf. Prognostics and Health Manage. PHM, Denver, CO, USA, 2008.

[13] J. Ajgl and M. Šimandl, "Particle based probability density fusion with differential Shannon entropy criterion," in Proc. 14th Int. Conf. Inf. Fusion ISIF, Chicago, IL, USA, 2011.

[14] O. Lanz, "An information theoretic rule for sample size adaptation in particle filtering," in Proc. 14th Int. Conf. Image Anal. and Process. (ICIAP), 2007, pp. 317-322.

[15] P. Skoglar, U. Orguner, and F. Gustafsson, "On information measures based on particle mixture for optimal bearings-only tracking," in Proc. IEEE Aerospace Conf. 2009, Big Sky, MT, USA, 2009.

[16] Y. Boers, J. N. Driessen, A. Bagchi, and P. K. Mandal, "Particle filter based entropy," in Proc. FUSION 2010, Edinburgh, U.K., 2010.

[17] T. M. Cover and J. A. Thomas, "Entropy, relative entropy and mutual information," in Elements of Information Theory. New York, NY, USA: Wiley, 1991, ch. 2.

[18] U. Orguner, "Entropy calculation in particle filters," in Proc. Signal Processing and Commun. Applicat. Conf., Antalya, Turkey, 2009.

[19] M. Orchard, M. Lacalle, B. Olivares, M. Cerda, and J. Silva, "Information-theoretic measures and sequential Monte Carlo methods for detection of regeneration phenomena in the degradation of energy storage devices," in Proc. 2013 Prognostics and System Health Manage. Conf., Milano, Italy, 2013

Marcos E. Orchard (M'06) is Associate Professor at the Electrical Engineering Department, University of Chile. His current research interest is real-time frameworks for fault diagnosis and failure prognosis, with applications to battery management systems, the mining industry, and finance. 
Matías S. Lacalle received the B.Sc. degree in electrical engineering from Universidad de Chile in 2013.

His research interests include health management of energy storage devices, renewable energy, and automatic control.

Benjamín E. Olivares received the B.S. and M.Sc. degrees in electrical engineering from Universidad de Chile, Santiago, in 2011 and 2012, respectively.

His research interests include prognostics and health management for energy storage devices based on optimal and suboptimal Bayesian algorithms.

Jorge F. Silva (S'06-M'09) is an Assistant Professor at the Electrical Engineering Department, University of Chile. His research interests include: estimation and detection, statistical signal processing, information theory, and statistical learning.

Rodrigo Palma-Behnke, (SM'04) is currently the Director of the Solar Research Center (SERC Chile). His research field is the planning and operation of electrical systems in competitive power markets and new technologies.
Pablo A. Estévez (M'98-SM'04) is a Full Professor with the EE Department, Universidad de Chile.

Dr. Estévez is the Vice President for Members Activities of the IEEE Computational Intelligence Society (CIS) for the period 2011-2014, and President-elect 2015 .

Bernardo Severino received his B.Sc. degree in electrical engineering from the University of Chile, 2011.

His main research interest is modeling and control of energy storage systems for microgrids.

Williams Calderon-Muñoz received the M.Sc. in Mechanical Engineering and $\mathrm{Ph} . \mathrm{D}$. in Aerospace and Mechanical Engineering from the University of Notre Dame, IN, USA.

$\mathrm{He}$ is currently an Assistant Professor in Mechanical Engineering in the Faculty of Physical and Mathematical Sciences, Universidad de Chile.

Marcelo Cortés-Carmona (M'90) is currently an Associate Professor in the Electrical Engineering Department at the Universidad de Antofagasta, Chile. His research interests include power systems planning, computational intelligence, and Lithium-Ion batteries. 